\title{
Resultados posoperatorios y satisfacción en pacientes operados de cirugía ortognática durante los años 2011-2018 en Hospital San José, Chile
}

\section{Post-operative results and satisfaction in patients operated of orthognathic surgery during the years 2011-2018 in Hospital San José, Chile}

\author{
Marcelo Mardones M. ${ }^{1,2,3}$, Rodrigo Bravo A. ${ }^{1,2,3}$, Renato Gunckel M., ${ }^{1,2,3}$, \\ Luis Córdova J. ${ }^{1,2,3}$, Coral Torres M. ${ }^{1}$.
}

\section{Resumen}

Introducción: Las dismorfosis dentofaciales (DDF) corresponden a un conjunto de alteraciones en la forma, posición o tamaño de los huesos maxilares. La cirugía ortognática es el tratamiento quirúrgico de elección para tratar este tipo de alteraciones, permitiendo la reposición de los maxilares y restitución de las funciones masticatorias, fonéticas y respiratorias, así como la estética facial. Objetivo: Describir resultados posoperatorios y el grado de satisfacción de los pacientes que optaron por la cirugía ortognática durante los años 2011-2018 por el equipo de Cirugía Maxilofacial del Hospital San José. Material y Método: Estudio retrospectivo, se evaluaron los protocolos operatorios de 44 pacientes operados de cirugía ortognática. Criterio de inclusión: pacientes mayores de 15 años con diagnóstico de DDF clases II y III no asociado a síndrome craneofacial. Se aplicó una encuesta a estos pacientes para conocer el grado de satisfacción con respecto a la cirugía ortognática efectuada, presencia de pérdida de sensibilidad neurológica y localización anatómica de ésta si la hubiese. Posteriormente, se relacionaron las variables género, edad del paciente al momento de la cirugía, pérdida de sensibilidad neurológica, tiempo de evolución posoperatorio y clase esqueletal con el nivel de satisfacción del paciente respecto a la cirugía ortognática. Resultados: Un 90,9\% de los pacientes está satisfecho con los resultados de la cirugía. No existe una relación estadísticamente significativa entre el grado de satisfacción con la cirugía ortognática y las variables, sexo, edad de operación, pérdida de sensibilidad neurológica (del nervio mentoniano, que da inervación sensitiva al tejido blando del mentón, labio inferior, encía por vestibular de incisivos, canino y primer premolar inferior), tiempo de evolución posterior a la cirugía y clase esqueletal. Conclusión: Existe un alto nivel de satisfacción en los pacientes intervenidos mediante cirugía ortognática. Las cinco variables analizadas no influyen en el nivel de satisfacción de los pacientes posterior a la cirugía ortognática.

Palabras clave: nivel de satisfacción del paciente, cirugía ortognática, dismorfosis dentofacial, prognatismo, retrognatismo.

\section{Abstract}

Introduction: Dentofacial deformities are a set of alterations in the shape, position or size of the maxillary bones. Orthognathic surgery is the surgical treatment of choice for this type of alterations, allowing the replacement of the maxillary and restitution of masticatory, phonetic and respiratory functions, as well as facial aesthetics. Aim: Describe post-operative results and level of satisfaction in patients who opted for orthognathic surgery during the years 2011-2018 by the Maxillofacial Surgery team of San José Hospital. Material and Method: Retrospective study, the surgical protocols of 44 patients who underwent orthognathic surgery were evaluated. Inclusion criteria: patients over 15 years old with diagnosis of DDF class II and III not associated with any craniofacial syndrome. A survey was applied to these patients to know the degree of satisfaction with respect to the orthognathic surgery
}

${ }^{1}$ Equipo Cirugía Maxilofacial, Servicio de Cirugía, Hospital San José. Santiago, Chile. 2Departamento de Cirugía y Traumatología Bucal y Maxilofacial, Facultad Odontología, Universidad de Chile. Santiago, Chile. ${ }^{3}$ Departamento de Cirugía Maxilofacial Clínica Las Condes. Santiago, Chile.

Los autores declaran no tener conflictos de interés.

Recibido el 23 de enero de 2020. Aceptado el 24 de abril de 2020.

Correspondencia: Marcelo Mardones M. San José 1196 , Independencia. Santiago, Chile. Email: drmardones@gmail.com 
carried out, the presence of loss of neurological sensitivity and its anatomical location, if any. Subsequently, the variables gender, patient age at the time of surgery, loss of neurological sensitivity, time of postoperative evolution and skeletal class were related to the level of satisfaction of patients with orthognathic surgery. Results: $90.9 \%$ of patients are satisfied with the results of the surgery. There is no statistically significant relationship between the level of satisfaction patients with orthognathic surgery and the variables, gender, age at the time of surgery, loss of neurological sensitivity (of the mentonian nerve, that gives sensitive innervation to the soft tissue of the chin, lower lip, gum by vestibular of incisors, canine and first lower premolar), time of postoperative evolution and skeletal class. Conclusion: There is a high level of satisfaction in patients undergoing orthognathic surgery. The five variables analyzed do not influence the level of patient satisfaction after orthognathic surgery.

Keywords: satisfaction patients, orthognathic surgery, dentofacial deformities, prognathism, retrognathism.

\section{Introducción}

Las dismorfosis dentofaciales (DDF) se caracterizan por la falta de armonía entre la cara y las estructuras óseas y dentales, generando un impacto negativo en el rostro, estética y equilibrio del sistema estomatognático ${ }^{1}$. Éstas no sólo afectan los aspectos oclusales y funcionales del sistema estomatognático, sino que también perjudican el bienestar psicosocial y estético de los pacientes, es decir, todos los componentes de la calidad de vida, alterándola significativamente ${ }^{2,3}$.

Las dismorfosis dentofaciales según su presentación clínica e imagenológica se clasifican, a modo general, en DDF de clase II y DDF de clase III. Las DDF de clase II son aquellas dismorfosis faciales en donde la relación sagital entre los maxilares y la mandíbula se encuentra en una posición posterior o distal respecto al maxilar superior determinando un tipo de perfil facial de característica convexa. Este tipo de dismorfosis tiene una fuerte asociación a problemas oclusales, respiratorios y articulares $^{1}$. Las DDF de clase III son aquellas dismorfosis faciales en donde la relación sagital entre los maxilares y la mandíbula se encuentra en una posición anterior respecto al maxilar superior, determinando un tipo de perfil facial de característica cóncavo. Este tipo de dismorfosis tiene una fuerte asociación a problemas oclusales, fonoarticulatorios y articulares ${ }^{1,3}$.

La cirugía ortognática es un tratamiento quirúrgico que permite la reposición de los maxilares y restitución de las funciones masticatorias, fonéticas y respiratorias, así como la estética facial de estos pacientes, mejorando de esta manera su calidad de vida ${ }^{4,5}$ (Figuras 1 y 2 ).
Esta cirugía corresponde a las osteotomías de los maxilares y de la unidad esqueletal mentoniana, determinando que sea, según la característica y necesidad de los casos, única, doble o triple de acuerdo a las estructuras anatómicas osteotomizadas $^{6}$. El impacto de este tipo de cirugía es alto, debido a que sus resultados comprometen la funcionalidad del sistema estomatognático y la imagen que tendrá el paciente de sí mismo en un futuro. Por este motivo es importante conocer los resultados posoperatorios y el nivel de satisfacción de los pacientes que ya fueron intervenidos mediante cirugía ortognática.

\section{Objetivo}

El objetivo de nuestra investigación es describir resultados posoperatorios y el grado de satisfacción de los pacientes intervenidos mediante cirugía ortognática durante los años 2011 al 2018 por el equipo de Cirugía Maxilofacial del Hospital San José y, además, determinar si existe una relación entre las variables sexo, edad al momento de la operación, pérdida de sensibilidad neurológica, tiempo de evolución posterior a la cirugía y clase esqueletal, con el grado de satisfacción de la cirugía por parte del paciente.

\section{Material y Método}

Se realizó un estudio retrospectivo de los casos registrados en la base de datos electrónica del Servicio de Cirugía Maxilofacial del Hospital San José. Para llevar a cabo esta 
investigación, se revisó la lista de pacientes operados de cirugía ortognática durante los años 2011-2018 por el equipo de Cirugía Maxilofacial. Incluimos en este estudio un total de 44 pacientes. Los criterios de inclusión fueron pacientes mayores de 15 años con diagnóstico de DDF clases II y III no asociado a síndrome craneofacial. Se revisó el protocolo operatorio y fueron consignadas las siguientes variables: género, edad en la que fue sometido a la cirugía, tiempo de evolución (definido como tiempo desde que fue sometido a la cirugía hasta ser contactado), clase esqueletal, tipo de cirugía, necesidad, motivo y tipo de reoperación en aquellos casos que fue necesario.

Los pacientes fueron contactados por vía telefónica para la aplicación de una encuesta de tres preguntas confeccionada por los autores, $y$ así registrar las siguientes variables: presencia o ausencia de pérdida de sensibilidad neurológica, región anatómica de pérdida de sensibilidad y grado de satisfacción con la cirugía. Para validar este instrumento se aplicó la validez de apariencia la cual no supone un concepto estadístico, debido a que el instrumento (cuestionario) es evaluado previamente por un grupo de expertos y otro de entrevistados, dependiendo así de los juicios de éstos ${ }^{7,8}$.

Para evaluar la validez de apariencia se conforma un grupo de jueces, por lo general expertos que determinan si en su concepto el instrumento en apariencia mide las cualidades deseadas, y otro grupo de personas que van a ser entrevistadas. La importancia de esta forma de validez está en la aplicabilidad y en la aceptabilidad del instrumento a ser aplicado ${ }^{8}$. De esta manera, al aplicar la validez aparente al instrumento, se discutieron las preguntas y alternativas conflictivas por dos expertos cirujanos maxilofaciales y el grupo piloto entrevistado, modificándose el lenguaje y el planteamiento de las alternativas. Se aplicó así la encuesta por vía telefónica, primero a un grupo piloto de seis pacientes del total de pacientes
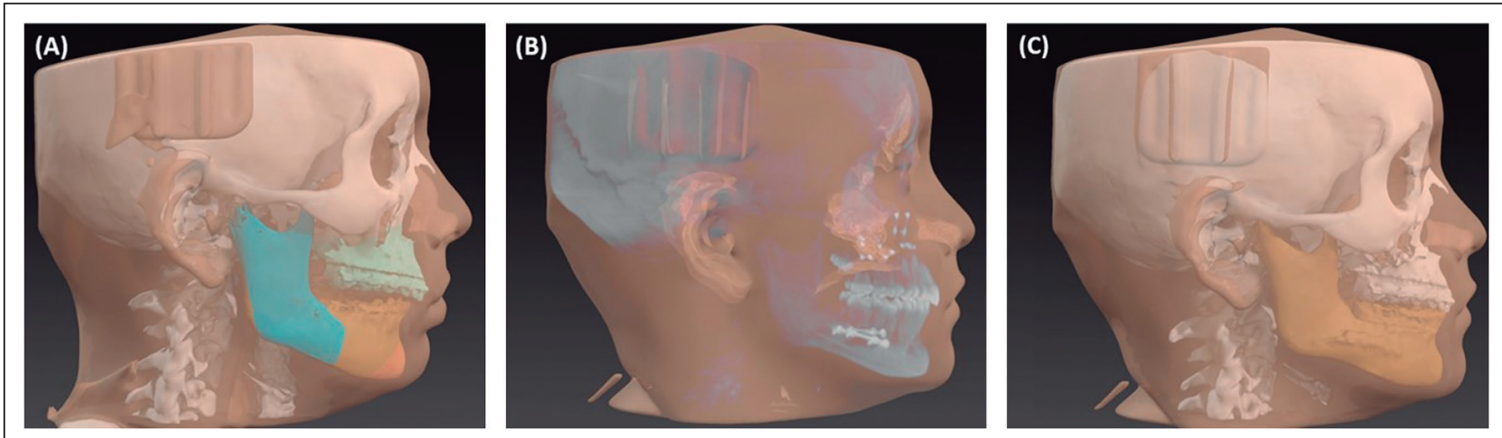

Figura 1. Paciente con dismorfosis dentofacial clase II. (A) Preoperatorio. (B) Posoperatorio con instalación de placas y tornillos de osteosíntesis. (C) Posoperatorio final.

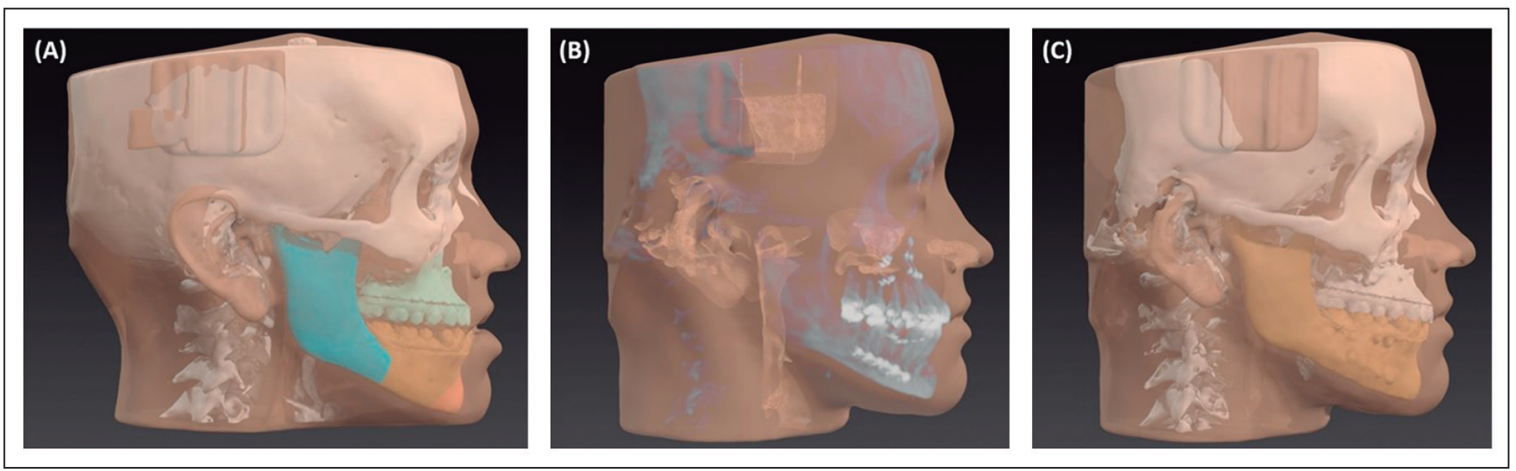

Figura 2. Paciente con dismorfosis dentofacial clase III. (A) Preoperatorio. (B) Posoperatorio con instalación de placas y tornillos de osteosíntesis. (C) Posoperatorio final. 
previamente seleccionados, para evaluar la capacidad de respuesta, el aspecto lingüístico y gramatical, la claridad y factibilidad de aplicar el instrumento y el tiempo aproximado requerido de colaboración por cada voluntario. Con estos resultados se discutieron las preguntas posiblemente conflictivas, obteniendo así la estructura final de la encuesta (Figura 3).

De acuerdo a los resultados obtenidos relacionamos el grado de satisfacción del paciente (satisfecho o no satisfecho) con la cirugía y las siguientes variables: género, edad del paciente al momento de la cirugía (menor o igual a 25 años; mayor a 25 años), pérdida de sensibilidad neurológica, tiempo de evolución posoperatorio (menor o igual a 2 años; mayor a 2 años), y clase esqueletal (clase II; clase III). Los resultados fueron analizados mediante el software estadístico GraphPad Prism, por medio de un análisis bivariado, mediante la prueba de proporciones Chi-cuadrado $\left(\chi^{2}\right)$ para analizar los datos obtenidos. Un valor de $\mathrm{p}<0,05$ fue considerado significativo, con un intervalo de confianza del 95\%.

\section{Resultados}

De los 44 pacientes considerados en el estudio, un $70,45 \%$ correspondió a mujeres y un $29,54 \%$ a hombres con una edad promedio de 23 años que optaron a la cirugía,

\section{Cuestionario de resultados posoperatorios y satisfacción cirugía ortognática}

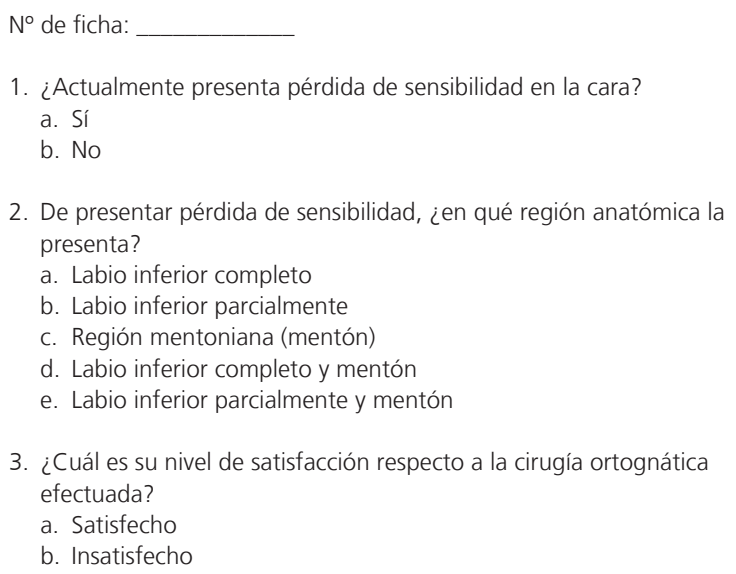

1. ¿Actualmente presenta pérdida de sensibilidad en la cara? a. Sí

b. No

2. De presentar pérdida de sensibilidad, ¿en qué región anatómica la presenta?

a. Labio inferior completo

b. Labio inferior parcialmente

c. Región mentoniana (mentón)

d. Labio inferior completo y mentón

e. Labio inferior parcialmente y mentón

3. ¿Cuál es su nivel de satisfacción respecto a la cirugía ortognática efectuada?

a. Satisfecho

b. Insatisfecho

Figura 3. Cuestionario de resultados posoperatorios y satisfacción en cirugía ortognática aplicado en los pacientes. un $77,27 \%$ menores o igual a 25 años y un $22,72 \%$ mayores a 25 años. La mayoría de los pacientes tratados presentaban una clase esqueletal tipo III $(70,45 \%)$, mientras que el tipo de cirugía más realizado fue cirugía triple $(59,1 \%)$ (osteotomía realizada en tres unidades esqueletales: ambos maxilares y la unidad esqueletal mentoniana) (Tabla 1). El $40,9 \%$ de los pacientes presentó un tiempo de evolución de la cirugía menor o igual a dos años, mientras que un $59,1 \%$ presentó un tiempo de evolución superior a los dos años. Un 31,8\% de los pacientes fue re-operado, siendo el retiro de placas el principal motivo de ésta (Tabla 1). Con respecto a la pérdida de sensibilidad posterior a la cirugía, un $84,1 \%$ presentó pérdida de sensibilidad (Tabla 1), siendo la región mentoniana la más predominante (57\%) (Tabla 2).

En relación al grado de satisfacción de los pacientes respecto a la cirugía ortognática, un $90,9 \%$ está satisfecho con los resultados de la cirugía, mientras que un 9,09\% se reportó insatisfecho (Tabla 1). Al relacionar las variables grado de satisfacción de los pacientes con respecto a la cirugía ortognática según género, se obtuvo que un $61,36 \%$ de mujeres estaba satisfecha con la cirugía, y un menor porcentaje $(9,09 \%)$ insatisfecha, comparado con un $29,54 \%$ de hombres satisfechos, mientras que no se reportaron hombres insatisfechos con ésta (Figura 4).

De acuerdo a la edad en que fueron sometidos a la cirugía, un 9,57\% de los pacientes mayores de 25 años estaba satisfecho con la cirugía y un 2,27\% insatisfecho. Mientras que un $70,45 \%$ de pacientes menores o iguales a 25 años estaba satisfecho con la cirugía y un $6,81 \%$ insatisfecho con ella (Figura 5).

De los pacientes con pérdida de sensibilidad un $75 \%$ estaba satisfecho con la cirugía y un 9,09\% insatisfecho. De los que no tuvieron pérdida de sensibilidad, un 15,9\% estaba satisfecho y no se reportaron pacientes insatisfechos (Figura 6).

En pacientes con un tiempo de evolución posterior a la cirugía menor o igual a 2 años, un $36,36 \%$ estaba satisfecho con la cirugía, mientras que un $4,54 \%$ insatisfecho. Por el contrario, aquellos pacientes con un tiempo de evolución mayor a 2 años, el 54,54\% estaba satisfecho y el 4,54\% insatisfecho (Figura 7). 


\begin{tabular}{|c|c|c|c|}
\hline Variables & & $\mathbf{n}$ & $\%$ \\
\hline \multirow[t]{2}{*}{ Sexo } & Femenino & 31 & 70,45 \\
\hline & Masculino & 13 & 29,54 \\
\hline \multirow[t]{2}{*}{ Edad de operación } & Menor o igual a 25 años & 33 & 77,27 \\
\hline & Mayor a 25 años & 11 & 22,72 \\
\hline \multirow[t]{2}{*}{ Clase esqueletal } & Clase II & 13 & 29,55 \\
\hline & Clase III & 31 & 70,45 \\
\hline \multirow[t]{3}{*}{ Tipo de cirugía } & Triple & 26 & 59,1 \\
\hline & Bimaxilar & 12 & 27,3 \\
\hline & Monomaxilar & 6 & 13,6 \\
\hline \multirow[t]{2}{*}{ Tiempo de evolución posquirúrgico } & Menor o igual a 2 años & 18 & 40,9 \\
\hline & Mayor a 2 años & 26 & 59,1 \\
\hline \multirow[t]{2}{*}{ Reoperación } & Sí & 14 & 31,8 \\
\hline & No & 30 & 68,2 \\
\hline \multirow[t]{5}{*}{ Motivo de reoperación } & Retiro de placas & 6 & 42,85 \\
\hline & Genioplastía & 2 & 14,28 \\
\hline & Septoplastía & 1 & 7,14 \\
\hline & Avance maxilar & 2 & 14,28 \\
\hline & Avance mandibular & 3 & 21,43 \\
\hline \multirow[t]{2}{*}{ Pérdida de sensibilidad } & Sí & 7 & 84,1 \\
\hline & No & 7 & 15,9 \\
\hline \multirow[t]{2}{*}{ Nivel de satisfacción con la cirugía } & Satisfecho & 40 & 90,9 \\
\hline & Insatisfecho & 4 & 9,09 \\
\hline
\end{tabular}

\section{Tabla 2. Región de pérdida de sensibilidad neurológica}

\begin{tabular}{lrr}
\hline Región & n & \% \\
\hline Labio inferior completo & 4 & 11 \\
\hline Labio inferior parcial & 3 & 8 \\
\hline Mentón & 21 & 57 \\
\hline Labio inferior completo y mentón & 6 & 16 \\
\hline Labio inferior parcial y mentón & 3 & 8 \\
\hline
\end{tabular}

Pacientes clase III esqueletal, un 65,9\% estaba satisfecho con la cirugía, mientras que un $4,54 \%$ insatisfecho. De los pacientes clase II, un $25 \%$ estaba satisfecho y un $4,54 \%$ no satisfecho con la cirugía (Figura 8).

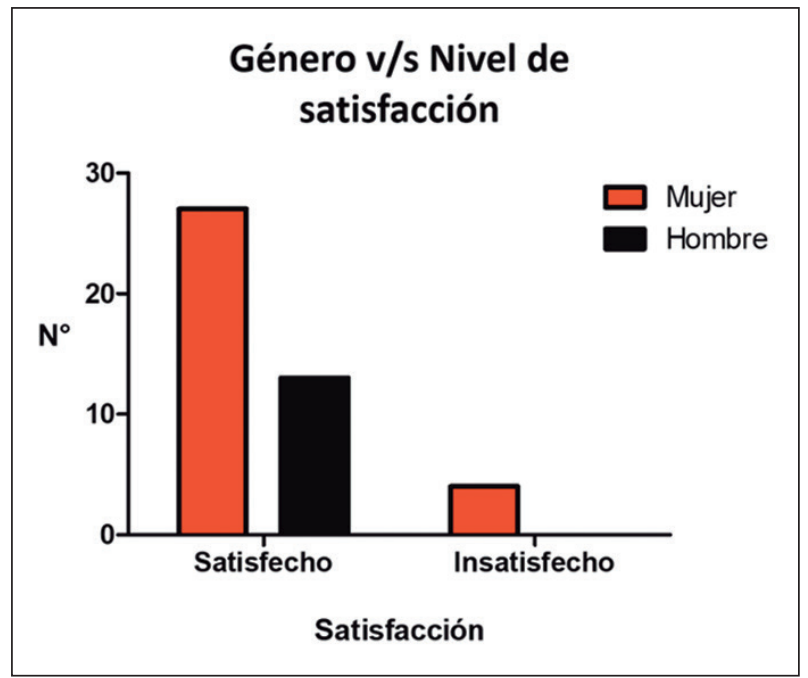

Figura 4. Nivel de satisfacción de cirugía ortognática según género. 


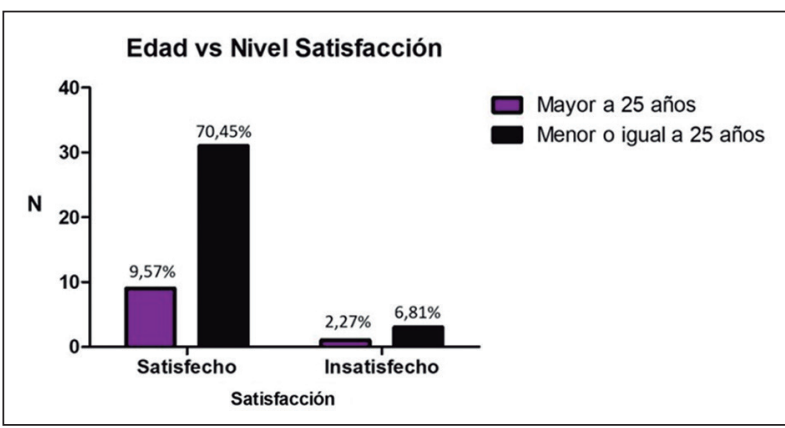

Figura 5. Nivel de satisfacción de cirugía ortognática según edad de operación del paciente.

Figura 6. Nivel de satisfacción de cirugía ortognática según pacientes que presentaron o no presentaron pérdida de sensibilidad neurológica.
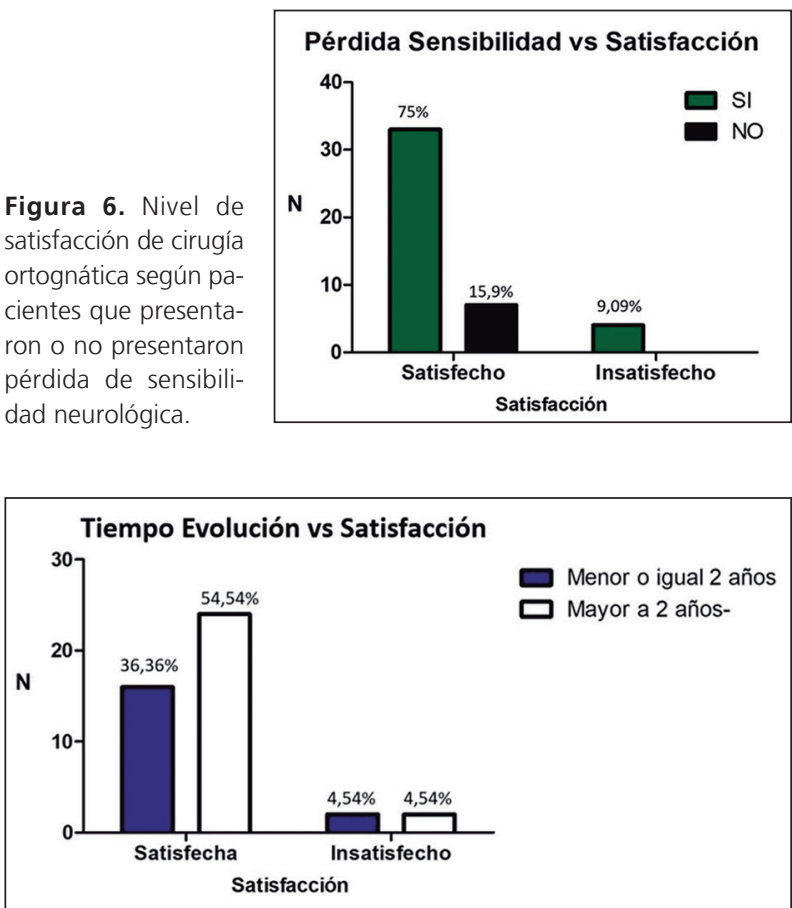

Figura 7. Nivel de satisfacción de cirugía ortognática según tiempo de evolución posterior a cirugía ortognática.

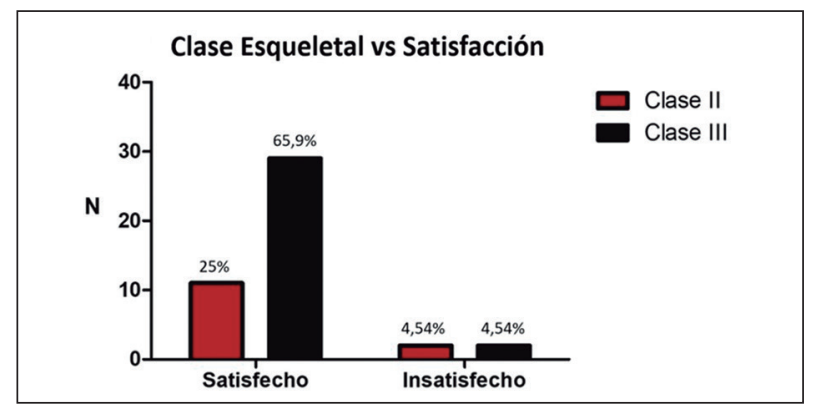

Figura 8. Nivel de satisfacción de cirugía ortognática según tipo de clase esqueletal.
Según el análisis de las variables estudiadas por medio de la prueba de proporciones Chi-cuadrado $\left(\chi^{2}\right)$, no hay una relación estadísticamente significativa entre el grado de satisfacción con la cirugía ortognática y las variables, sexo, edad de operación, pérdida de sensibilidad neurológica, tiempo de evolución posterior a la cirugía y clase esqueletal. De manera que éstas no influyen en el nivel de satisfacción de la cirugía del paciente. Sin embargo, de los pacientes operados, el grado de satisfacción posterior a la cirugía ortognática es mayor que los pacientes insatisfechos.

\section{Discusión}

El objetivo principal de nuestro estudio es describir resultados posoperatorios y el grado de satisfacción de los pacientes intervenidos mediante cirugía ortognática durante los años 2011 a 2018 por el equipo de Cirugía Maxilofacial del Hospital San José y, además, determinar si existe una relación entre las variables sexo, edad al momento de la operación, pérdida de sensibilidad neurológica, tiempo de evolución posterior a la cirugía y clase esqueletal con el grado de satisfacción de la cirugía por parte del paciente. De acuerdo a nuestro estudio, el nivel de satisfacción de los pacientes con respecto a la cirugía ortognática es mayor $(90,9 \%)$, en comparación con aquellos pacientes que estaban insatisfechos con los resultados de la cirugía, coincidiendo así con los resultados de investigaciones actuales ${ }^{3,6,7}$. Estudios de AlKharafi y cols. ${ }^{7}$ y Posnick y cols. ${ }^{8}$ indican tasas de satisfacción superiores al 80\% con los procedimientos ortognáticos. Mientras que Bock y cols. ${ }^{9}$ dan a conocer un grado de satisfacción posterior a la cirugía basado en cuestionarios, variando entre un $87 \%-100 \%$ de los pacientes con un grado alto de satisfacción.

De un total de 44 pacientes, el 70,45\% correspondió a mujeres y un $29,54 \%$ a hombres, lo que demuestra que existe una mayor demanda por parte de las mujeres con DDF para la realización de cirugía ortognática. Una posible explicación para esta diferencia entre los géneros podría ser que las mujeres están más preocupadas sobre su apariencia y funcionalidad maxilomandibular que los hombres ${ }^{4,10}$. La edad promedio en que los pacientes fueron 
sometidos a cirugía ortognática fue de 23 años, predominantemente los pacientes con una edad menor o igual a 25 años, coincidiendo así con estudios de Huang y cols. ${ }^{11}$ y de Alves y Silva ${ }^{5}$. La región anatómica con mayor pérdida de sensibilidad neurológica reportada correspondió a la mentoniana, la cual fue recuperada dentro de un periodo de 6 meses. Así mismo, estudios de Sadat-Marashi y cols. indicaron mayor frecuencia de hipoestesia transitoria en el área mentoniana ${ }^{10}$.

Por otra parte, el estudio realizado reporta que las variables sexo, edad, pérdida de sensibilidad neurológica, tiempo de evolución posterior a la cirugía y clase esqueletal no influyen en el nivel de satisfacción de los pacientes operados de cirugía ortognática, debido a que no existe una relación estadísticamente significativa entre estas cinco variables y el grado de satisfacción. Emadian Razvadi y cols. ${ }^{4}$ coinciden con nuestro estudio, reportando que el género no influye en el grado de satisfacción de los pacientes respecto a la cirugía ortognática. Reportaron un porcentaje mayor de hombres satisfechos, sin embargo, nuestro estudio reportó un mayor porcentaje de mujeres satisfechas con el tratamiento realizado, coincidiendo con el estudio de Finlay y cols. ${ }^{12}$.

Pacientes que fueron operados a una edad menor o igual a 25 años reportaron mayores niveles de satisfacción que pacientes mayores de 25 años, no obstante, la edad no tiene una relación estadísticamente significativa en el nivel de satisfacción de cirugía ortognática, lo que coincide con resultados similares en el estudio de Emadian Razvadi y cols. ${ }^{4}$ y Larsen y cols. ${ }^{13}$. Pacientes que presentaron pérdida de sensibilidad neurológica en alguna región anatómica de su cara, refirieron un porcentaje mayor de nivel de satisfacción con respecto a la cirugía ortognática, con respecto a pacientes que no tuvieron pérdida de sensibilidad. Estudios de Rustemeyer y cols. ${ }^{14}$ y Zamboni y cols. ${ }^{15}$ indicaron que la presencia de problemas de sensibilidad no generó problemas de satisfacción en los pacientes, manteniéndose alta como en nuestro estudio. Sin embargo, otros estudios ${ }^{3,12,16}$ reportaron que pacientes con tendencia a experimentar insatisfacción con la cirugía, se relacionaba con pérdida de sensibilidad de alguna región anatómica, junto con dificultad para masticar e inflamación.
Emadian-Razvadi y cols. ${ }^{4}$ informaron que los problemas postoperatorios pueden afectar la satisfacción postquirúrgica temprana a los tres meses y su efecto desaparecería entre 6 y 12 meses.

Pacientes con un tiempo de evolución posterior a la cirugía ortognática mayor a 2 años, estaban más satisfechos que aquellos que llevaban menos de 2 años de operados. Si bien en este estudio no se reportó una relación estadísticamente significativa entre estas variables, estudios indican que, si el nivel de satisfacción se mide solo unos pocos meses después de la cirugía ortognática, se reporta cierta insatisfacción, posiblemente debido a los efectos secundarios persistentes ${ }^{10}$. Sin embargo, con el paso del tiempo, la satisfacción mejora y se ha encontrado que es alta un año después de la cirugía $^{12}$. A pesar del hecho de que la mayoría de los estudios informaron el mismo hallazgo, no hay consenso sobre la relación entre el índice de satisfacción y el tiempo de evaluación ${ }^{10}$.

Este estudio reportó que pacientes con DDF clase III están más conformes con la cirugía ortognática que los pacientes clase II. En particular, la dismorfosis dentofacial de clase III presentan mayores alteraciones estéticas y funcionales que pueden causar problemas psicológicos e interpersonales, en relación a las DDF de clase $\mathrm{II}^{14}$. Nuestros resultados revelaron un $65,9 \%$ de satisfacción en este tipo de pacientes, en comparación con un 25\% de satisfacción en pacientes DDF clase II. Estudios de Rustemeyer y cols. ${ }^{14}$ y Corso y cols. ${ }^{17}$ reportaron un $77,9 \%$ de satisfacción con la cirugía en pacientes clase III, lo que coincide con nuestro estudio.

\section{Conclusión}

Los resultados de este estudio demostraron que existe un alto nivel de satisfacción en los pacientes sometidos a cirugía ortognática. No existe relación estadísticamente significativa entre las variables sexo, edad, pérdida de sensibilidad neurológica, tiempo de evolución posterior a la cirugía y clase esqueletal con respecto al nivel de satisfacción de los pacientes. De esta manera, estas cinco variables estudiadas, no influyen en el grado de satisfacción de los pacientes posterior a la cirugía ortognática. 
La cirugía ortognática es un procedimiento quirúrgico que resuelve de manera satisfactoria las alteraciones funcionales y estéticas de las dismorfosis faciales, determinando un alto grado de satisfacción en los pacientes que reciben este tratamiento.

\section{Agradecimientos}

Agradecimientos al Dr. Pablo Romero R. que colaboró en el análisis estadístico de las variables, permitiendo la interpretación de los resultados.

\section{Bibliografía}

1. Alanko O, Tuomisto MT, Peltomäki T, Tolvanen M, Soukka T, Svedström-Oristo AL. A longitudinal study of changes in psychosocial well-being during orthognathic treatment. Int J Oral Maxillofac Surg 2017;46:1380-1386. doi:10.1016/j.ijom.2017.05.004

2. Silva I, Suska F, Cardemil C, Rasmusson L. Stability after maxillary segmentation for correction of anterior open bite: a cohort study of 33 cases. J Craniomaxillofac Surg 2013;41:e154-e158. doi:10.1016/j.jcms.2012.12.003

3. Kufta K, Peacock ZS, Chuang SK, Inverso G, Levin LM. Components of Patient Satisfaction After Orthognathic Surgery. J Craniofac Surg 2016;27:e102-e105. doi:10.1097/ SCS.0000000000002318

4. Emadian Razvadi ES, Soheilifar S, Esmaeelinejad $M$, Naghdi N. Evaluation of the Changes in the Quality of Life in Patients Undergoing Orthognathic Surgery: A Multicenter Study. J Craniofac Surg 2017;28:e739-e743. doi:10.1097/ SCS.0000000000003887

5. Alves e Silva AC, Carvalho RA, Santos Tde S, Rocha NS, Gomes AC, de Oliveira e Silva ED. Evaluation of life quality of patients submitted to orthognathic surgery. Dental Press J Orthod 2013;18:107-14. doi: 10.1590/s2176-94512013000500018.

6. Miguel JA, Palomares NB, Feu D. Life-quality of orthognathic surgery patients: the search for an integral diagnosis. Dental Press J Orthod 2014;19:12337.

7. AlKharafi L, AlHajery D, Andersson L. Orthognathic surgery: pretreatment information and patient satisfaction. Med Princ Pract 2014;23:218-24.

8. Posnick JC, Wallace J. Complex orthognathic surgery: assessment of patient satisfaction. J Oral Maxillofac Surg 2008;66:934-42.

9. Bock JJ, Maurer P, Fuhrmann RA. The importance of temporomandibular function for patient satisfaction following orthognathic surgery. J Orofac Orthop 2007;68:299.

10. Sadat-Marashi Z, Scolozzi P, Antonarakis GS. Perceptions of Young Adults Having Undergone Combined Orthodontic and Orthognathic Surgical Treatment: Grounded Theory Approach. J Oral Maxillofac Surg 2015;73:2391-8.

11. Huang S, Chen W, Ni Z, Zhou Y. The changes of oral health-related quality of life and satisfaction after surgery-first orthognathic approach: a longitudinal prospective study. Head Face Med. 2016;12:2. doi:10.1186/s13005-015-0098-1.

12. Finlay PM, Moos SF, Atkinson JM. Orthognathic surgery: patient expectations; psychological profile and satisfaction with outcome. Br J Oral Maxillofac Surg 1995;33:9-14.

13. Larsen MK, Thygesen TH. Orthognathic Surgery: Outcome in a Facebook Group. J Craniofac Surg 2016;27:350-5.

14. Rustemeyer J, Eke Z, Bremerich A. Perception of improvement after orthognathic surgery: the important variables affecting patient satisfaction. Oral Maxillofac Surg 2010;14:155-62.

15. Zamboni R, de Moura FRR, Brew MC, Rivaldo EG, Braz MA, Grossmann E, et al. Impacts of Orthognathic Surgery on Patient Satisfaction, Overall Quality of Life, and Oral Health-Related Quality of Life: A Systematic Literature Review. Int J Dent 2019;2019:2864216.

16. Pachêco-Pereira C, Abreu LG, Dick BD, De Luca Canto G, Paiva SM, Flores-Mir C. Patient satisfaction after orthodontic treatment combined with orthognathic surgery: A systematic review. Angle Orthod 2016;86:495-508.

17. Corso PF, Oliveira FA, Costa DJ, Kluppel LE, Rebellato NL, Scariot R. Evaluation of the impact of orthognathic surgery on quality of life. Braz Oral Res 2016;30. pii: S1806-8324201600010020. 